\title{
Application of Data Mining Technology in Intelligent Electronic Commerce Search and Recommendation
}

\author{
Xue Bai * \\ Department of Economic Management \\ Guangdong Polytechnic \\ Foshan, China \\ chenxiaoyunld@163.com \\ * Corresponding Author
}

\begin{abstract}
Personalized recommendation technology is the core and key technology in the electronic commerce recommendation system. Data mining technology can help decision makers to find out the potential relationship between the data and find out the potential relationship between the data. This paper firstly analyzes the application of intelligent electronic commerce search and personalized recommendation, and presents application of data mining technology and intelligent agent in e-commerce recommendation system. Finally, the paper presents the application of data mining technology in intelligent ecommerce search and recommendation.
\end{abstract}

Keywords- Data Mining; Electronic Commerce; Personalized Recommendation; Search; Intelligent

\section{INTRODUCTION}

Electronic commerce, source from English electronic commerce, abbreviated as EC, refers to is the use of simple, fast, low-cost electronic means of communication, the sale of the two sides are not met for various business activities. Its content includes two aspects: one is the electronic way; the two is the business activity [1]. E-commerce can be accomplished through a variety of electronic communication, but now people are discussing the electronic commerce is mainly based on EDI (electronic data exchange) and INTER to complete. Especially with the increasing maturity of INTERNET technology, the real development of e-commerce will be built on INTERNET technology, so it is also referred to as IC.

Personalized recommendation technology is the core and key technology in the electronic commerce recommendation system and the main representative is collaborative filtering technology. The technology does not focus on the performance of the content, but according to the user's information structure model to confirm the neighbor users. Through the user's evaluation of the content of the user, and it is the user's interest in a similar degree after relying on the user's interest to recommend to the user.

The macro level of e-commerce is a large enterprise, research institutions, media organizations, the need for professional, the latest information, the information provided to the user to help users make decisions and research, and provide such services will require a strong information acquisition and strong information system support. Micro level e-commerce user groups are mainly to price as a link to the small businesses and general consumers, the site's main business focus on ensuring the normal operation of small businesses and consumers can easily access to price information.

In recent years, e-commerce services have been fully covered in all aspects of commercial economy: whether it is the national economy in the manufacturing field, or the service industry in the field of circulation, whether business applications, personal applications, or government procurement [2]. More and more large and small businesses have finally seen the benefits of ecommerce, whether it is the official independent of the official e-commerce platform, or the use of the third party electronic commerce platform, so that e-commerce penetration will continue to maintain rapid growth.

The cooperative work of data mining and data warehouse not only improves the efficiency and ability of data mining, but also ensures the integrity and integrity of the data sources in data mining. Moreover, it has become a very important aspect of data warehouse application. Based on the brief introduction of data warehouse, data mining technology and the concept of electronic commerce, this paper analyzes the application of data warehouse and data mining technology in the intelligent electronic commerce search and recommendation.

\section{RESEARCH ON THE APPLICATION OF INTELLIGENT ELECTRONIC COMMERCE}

Network business platform to the backbone of talent than those simply opened the shop B2C e-commerce business talent, but those who can contact each B2C shop owner and various $\mathrm{B} 2 \mathrm{C}$ online communication, can find B2C shop product demand, can use Park supply chain support the ability to quickly organize relevant product line to meet the demand of $\mathrm{B} 2 \mathrm{C}$ network of talent. On the other, the large scale target customers intends to use Haitian in the influence of the traditional brand channels to carry out net goods production business cooperation to obtain basic net goods business orders and medium scale target customers are actively looking for medium scale advantage B2C shop, taking advantage of production services can 
force to the service of the shop brand development, small scale target customers, to high-quality product line resources supporting the discount conditions to attract the consignment Haitian company brand products.

Personalized customized information needs will be strong, the depth of personalized products become a necessity [3]. The emergence of the Internet, the popularity and development itself is on a traditional order of social and economic organization and individual liberation, and personality publicity and creativity play have a more favorable platform, also make the realization of consumer sovereignty with more effective technology base. In this regard, the demand for personalized customized information and personalized product demand will become the direction of development, consumers will participate in the product design and manufacturing process, to provide a variety of e-commerce activities for individual consumers, more personalized services than the traditional business, is the key factor to determine the success of the future.

E-commerce as an important industry in the modern service industry, the "sunrise industry, the green industry," said, with the characteristics of "three high", "Sanxin". "Three high" that the high level of human capital, high technology content and high added value, "Sanxin" is refers to the new technology, new formats and new ways. Stream of people, logistics, capital flow, information flow "four flow" is the core value chain of e-commerce. Ecommerce industry has the advantages of the market globalization, the transaction continuity, the low cost, and the intensive resources and so on. E-commerce in China's industry, agriculture, commerce, transportation, transportation, finance, tourism and urban and rural consumption and other fields of application has been expanded, increasing the level of application, is the formation and development of the real economy in-depth integration.

The focus of the electronic commerce of the mobile intelligent terminal will be transferred to the enterprise application. In recent years, with the rapid development of mobile communication technology, mobile technology and e-commerce are more closely related to the market economy, enterprises as the main body of its application in the adaptation of mobile users personalized needs, will be its main business activities to mobile business, so as to use the unique advantages of mobile e-commerce to achieve business growth, as is shown by equation (1).

$$
\tilde{Q}(m)=\operatorname{diag}[Q(m M), Q(m M+1), \cdots, Q(m M+M-1)]^{T}
$$

With the development of communication technology and networking technology, mobile Internet is showing a flourishing situation, more and more enterprises have put their eyes on the mobile electronic business, and a variety of applications are gradually entering people's eyes. Mobile e-commerce to create more business opportunities, but also for their own development and create more business opportunities; second because of mobile ecommerce is the combination of communication technology and electronic commerce, the two major areas of mobile e-commerce industry chain; new technology continues to be born and applied, will allow smart mobile terminal to bring more space for mobile e-commerce, but also a lot of problems to be solved at the same time.
Because the target user is different, it is decided that the intelligent agent is applied to the macro and micro electronic commerce service. Macro level e-commerce is the main service for large enterprises, research institutions and other information, which requires that the source of information must be extended to the entire industry and the special needs of the user to carry out information agents. The micro level of e-commerce services is relatively narrow, mainly in their own website to register the product information of small businesses, as is shown by equation (2) quotation information, to provide users with intelligent, personalized product price information [4].

$$
C(t)=\frac{E[B(t), B(-t)]}{E\left[B(t)^{2}\right]}=2^{2 H-1}
$$

Low information content customers, this kind of customer, including all non registered customers, registered but the low rate of account activity. Some of these casual customers are likely to be potential customers, and if the product is recommended in the initial period of their initial visit, it is likely that these potential customers will be transformed into a valued customer. Because the electronic commerce website does not have the master customer's basic information and the historical information, it needs to collect this kind of customer information and to make the recommendation in time.

The main information of the collection is access path, sequence pattern, dwell time, etc. The whole recommendation process from the customer into the site, with the behavior of the customer in the website, the recommendation system to the customer's classification more accurate, the more detailed, the recommendation results also have a from coarse to fine process. The key problem in the implementation of the recommendation system is to improve the real-time performance.

Data mining is to explore the large amount of data in accordance with the established business objectives, and to reveal the hidden rule of the law well to further its model of advanced and effective method of mass data processing. Data mining technology makes data warehouse decision support is the best tool, can help decision makers from commercial accumulation such as "mountain", potential such as "flood" of a large number of data mining hidden regularity, so as to support decision makers in time to make the right and scientific management decision-making.

Collaborative filtering technology is generally divided into two categories: user based collaborative recommendation and model based collaborative recommendation [5]. The former is to use statistical methods to obtain a similar interest in the neighbor users, and weighted neighbor users in a specific project evaluation score to predict the current user evaluation scores on the project, according to the score given recommendation. The latter is constructed by the historical data structure model, predict potential demand. Here are mainly based on the user based collaborative filtering recommendation system.

For the concept of data warehouse, we can understand from two levels: first, data warehouse is used to support decision-making, analysis oriented data processing, it is different from the existing operational database; secondly, 
data warehouse is the effective integration of multiple heterogeneous data sources. Artificial intelligence is to design and develop a variety of computer programs to simulate human thinking structure, reasoning and solving problems.

$$
(Z-\hat{Z})^{T}(Z-\hat{Z})=\min
$$

In E-commerce, the customer, market, sales, service and support information can be understood by the customer, market, sales, and service and support information. Provide quality personalized service to enhance customer loyalty. In the electronic commerce activities, the content of the website, title, reward programs, services and other aspects are likely to attract customers.

Building a professional network marketing platform, customers can fully understand the product's functionality and services through access to the company's website, through the DV video to simulate the entire warehouse service process. By and large domestic B2C, C2C cooperation platform, and it are remote sales presentation to potential network. Google, Baidu and other search engines for the key words for the ranking, and it is the promotion of intelligent warehouse service products. Through the establishment of the electricity suppliers to set up stalls, directly facing the electricity suppliers to demonstrate and promote the service.

\section{ANALYSIS ON THE APPLICATION OF DATA MINING TECHNOLOGY AND INTELLIGENT AGENT IN THE \\ ELECTRONIC COMMERCE RECOMMENDATION SYSTEM}

In the macro level, the information system provides the main body of the information system. The information system provides information service, so it is not necessary to carry out credit evaluation. And in the micro level intelligent agent of electronic commerce, not only need to according to the user's subjective comments for credit evaluation, also applied to the intelligent interactive evaluation based on orders, and thus more can reflect the operation of the online store, according to the objective data to explain the problem and evaluate the credit situation, to provide users with help.

Improve the hit rate, improve the electronic commerce website design through data mining technology, analysis of customer behavior and feedback behavior, e-commerce companies can more effectively optimize the structure of the site, improve the site's hit rate [6]. For example, through the association rules, for customer needs, adjust the structure of the site, the customer access to the related documents to be directly linked, so that customers can easily access to the page, increase the probability of customer visit again, as is shown by equation(4).

$$
f_{\max }=\pi / 2, q=\sqrt{2}
$$

Data warehouse has two major advantages: first, do not need to re compile the input (thing) processing system to build a structured environment, the output (decision support) processing into a new environment (data warehouse); two is the data warehouse to establish the concept model (logical data model), internal model (historical documents) and external model (data warehouse) three mode environment. In which the "multi - to -" maintenance of historical documents can be simplified to three "one to many" relationships under the mode of the model.

Production platform is network business platform business model can be the key to successful operation support platform; the traditional production chain with net goods production of many varieties, small batch, and short period of production economic benefit requirement is not high. Should therefore be appropriate adjustments, and the improvement of existing production chain, in the form of centralized + scattered re divided, every section of production chain modular deployment, shorten the module production process, increase the processing capacity, improve the collaborative ability among the modules, so that the production chain is fully functional and flexible, both to meet the needs of the traditional production business, and meet the production needs of net goods business, thus to build new production platform. The adjusted production chain can reduce the investment scale of fixed assets and logistics of the production unit, and can avoid the investment risk.

Mobile terminal software and hardware platform is difficult to unified one. With the development of mobile terminal equipment in recent years, the major operators have developed a variety of smart mobile devices, a wide variety of smart phones, tablet PCs, mobile watches are emerging, resulting in mobile terminal software and hardware devices ten points chaos, the operating system has emerged IOS, windows, Android and other good.

In the macro level intelligent agent of e-commerce, the need for all employees to fully participate in the enterprise information portal system, the system function is applied and explore, in the allowable range of customized information services, to ensure the smooth operation of the system [7]. The enterprise information portal system has the intelligence, and comes with the media to upgrade the function automatically, the user does not need to maintain. In the micro level of intelligent agents in the electronic commerce of the user has two parts, and consumers, online stores need to carry out regular maintenance and information updates, and consumers do not need to maintain.

$$
\sum_{b_{j}=1}^{M} q_{b_{j}}\left(y_{0}\right)=1
$$

With the increasingly fierce competition in ecommerce, as is shown by equation (5), the role of business intelligence in e-commerce operations will gradually become important; this point from foreign mature e-commerce sites can be seen. In foreign countries, the general e-commerce enterprises will have a special person responsible for data analysis and other business intelligence, such as business intelligence, as a strategic investment. From the current China's e-commerce industry, the real concern about data analysis and business intelligence is not much, but the lack of capital investment is the main reason for the enterprise.

But the importance of data for e-commerce enterprises is self-evident, e-commerce oriented business intelligence 
management system is based on the combination of intelligent management system and online decision support system, the integration of intelligent, integrated, coordinated, networking and online decision support to the traditional business computer management system, so that it can adapt to the modern business development trend, to provide better management environment and decision support.

Data warehouse is used to realize the storage and synthesis of decision subject data. Through extracting, cleaning, integration and transformation from the source database, and providing standard report and chart. Through various angles, we can construct the multi dimension data model, as is shown by equation (6) [8].

$$
X_{i, V}(m)=\bar{H}_{i} X_{i+1, V}(m), L \leq i \leq N-1
$$

Product list: according to the collected information of customers, through data analysis can get the customer the most interested commodity list, recommend to customers. The formation of professional technical team and it is the advantages and disadvantages of the focus on target customer. Analysis root cause, develop solutions, targeted training program effectiveness. Sales team focused on the network personnel to explore, develop, training and motivation, familiar with the electricity business network activity, the establishment of communication channels and ways to understand their needs, and recommend the level of service products.

In artificial intelligence, this field includes language processing, automatic theorem proving, intelligent data retrieval system, vision system, problem solving, artificial intelligence method and programming language. In the last 30 years, some computer systems with artificial intelligence have been established. For example, to solve differential equations, to design and analyze the integrated circuits, to synthesize human natural language, to control the intelligent computer system with different degrees of artificial intelligence.

The traditional electronic commerce website only realizes the function of the commodity trade, the sales system and the production system are separated each other, reducing the system's utilization rate and the use space. In customer management, people have too many limitations to the registration information acquisition, ignoring the user needs to change, the use of user information into a single text information reserves.

$$
(1-B)^{\delta}=\sum_{k=0}^{\infty}\left(\begin{array}{l}
\delta \\
k
\end{array}\right)(-1)^{k} B^{k}
$$

The theory and technology of intelligent (Agent) have been proposed in 1990s, it is a new research and development field, which is related to artificial intelligence, information retrieval, computer network, database, data mining, natural language processing and other fields of theory and technology, mainly artificial intelligence. However, to date, there is no unified and authoritative opinion on the theory, but many experts have a common feature in the definition of intelligent agents. Intelligent agents can imitate human social behavior through perception, learning, reasoning and action.

Collect customer information: This includes the personal attributes of the customer, the purchase of the historical records, web pages of the record, etc. Personal attributes can be obtained from the user registration form; of course, the premise is that the user must fill out the true and valid personal information [9]. Those in the leading ecommerce enterprises in the resources, brand, customer size and other aspects have a great advantage, the midpoint of the network that the foreign well-known e-commerce companies, there is a big gap.

As the two major trends of the development of electronic commerce, each site is limited in resources, the customer's demand is all round, so different types of Web sites in the form of strategic alliances will become an inevitable trend. Because of the many e-commerce sites, customers can easily switch between sites, so e-commerce sites should be able to access information to customers through the customer's browsing behavior, so as to understand customer loyalty, preferences and needs, quickly adjust the WEB page to meet customer needs. For example, customer needs can be presented to the relevant items, to facilitate customer selection.

\section{RESEARCH ON APPLICATION OF DATA MINING TECHNOLOGY IN INTELLIGENT ELECTRONIC COMMERCE SEARCH AND RECOMMENDATION}

As the number of network operators suddenly soared and it is far beyond the expectations of the market. However, for closely matching the warehousing service is almost blank. Most of the electric storage form still remains in the traditional logistics model: 1 . The network received orders after the plant on behalf of the goods; 2 . The small size of the hoard goods in the home, office or store; 3. Lease a small warehouse, let express company before to pick up pieces on behalf of. Electronic business intelligence warehouse: e-commerce businesses to complete the entire online sales transaction process, the goods receipt inspection warehousing, classified management, security, inspection delivery, customer information security and other links, collectively referred to as smart storage.

Time variant for decision needs, data warehouse data need to indicate the time parameter, and with the time to change, that is, with the time changes, constantly have new data content to add; constantly export and delete useless data content; continue to re integrated data.

Exhibition Center planning as the studio's supporting service platform, intends to the studio to provide a centralized display of its products or the ability of space, and as the studio and $\mathrm{B} 2 \mathrm{~B}$ network communication platform, B2B network operators can, through this platform to quickly find and organization to meet the market demand of the product development supporting units, as is shown by equation (8).

$$
\operatorname{Var}[X]=E\left[\left(X-\mu_{X}\right)\left(X-\mu_{X}\right)^{T}\right]=C_{X}
$$

This paper summarizes the application of artificial intelligence technology in electronic commerce from the aspects of the development of artificial intelligence and 
electronic commerce, the application of artificial intelligence technology in electronic commerce, and the application of data mining technology in Web. With the continuous development of electronic commerce and the continuous improvement of artificial intelligence technology, the integration of both in various fields and various levels will be more closely [10]. As a successful factor, the integration of e-commerce and artificial intelligence technology will become a key technology.

Business intelligence management system is a set of technologies, such as artificial intelligence, knowledge engineering, pattern recognition, machine learning, and data mining and so on. Through the integration of methods and technology integration, to achieve functional integration, the business intelligence management system not only has the function of information management, but also has the function of decision support, information management and business processing.

Agency is also a basic feature of intelligent agent technology, mainly refers to the ability of autonomous and coordination of intelligent agents. The performance of intelligent agent in the degree of automation, that is, the operation can leave the person or the agent of the intervention, can automatically use the agent communication protocol for information exchange, and the results of the retrieval information to the user, and manage the user's personal data and private directory. But the agent must be controlled in the system. When the other agent is put forward, only the agent can decide whether to accept or reject the request.

In the whole process of the electronic commerce intelligent recommendation system, the full range of safety control is the necessary means to ensure the normal operation of the system. Whether it is the privacy of user information, or the system robustness, security maintenance, are very important. We need not rain made mistakes, shortcomings of the further development and improvement of e-commerce at the present stage of intelligent recommendation system.

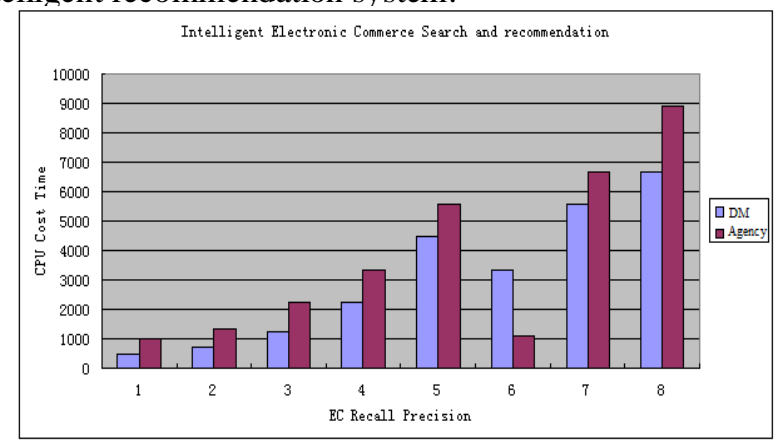

Figure 1. Application of Data Mining Technology in Intelligent Electronic Commerce Search and Recommendation.

Recommended system and production and manufacturing integration: users can order related products based on demand. Enterprises regularly collect user feedback, adjust the industrial structure, the implementation of personalized product production, user needs to guide the production and manufacturing.
Recommendation system and sales system integration: through the purchase of historical statistics, product demand analysis, according to the user needs to adjust the sales strategy.

From the process of real-time intelligent processing of E-Commerce orders can be seen, the real-time intelligent processing of E-Commerce orders from the customer facing layer processing and customer order delivery arrangements for two core processing, supporting these two core processing rule base and knowledge base is to achieve intelligent processing of data and knowledge base.

\section{SUMMARY}

Because of the abundant information resources in the field of electronic commerce, it provides a good foundation for the enterprise to implement data warehouse and data mining technology. At the same time, the data warehouse and data mining technology provide a strong technical support for e-commerce, and accelerate the development and popularization of electronic commerce. Data warehouse and data mining technology has become one of the most popular technology in the field of electronic commerce. Through the collation and induction of the source data, it can help decision makers to find the potential relationship between the data, and find the information hidden behind the data, not only can predict the customer's consumption trend and further market trend.

\section{REFERENCES}

[1] ZHANG Xudong, "The Research on the Electronic Commerce of Independent Trust System in China", JCIT, Vol. 8, No. 7, pp. 113 121,2013

[2] Xiangwei $\mathrm{Mu}$, Yan Chen, Yan Cao, Yan Li, "Personalized Recommendation System Modeling in Semantic Web", AISS, Vol. 5, No. 2, pp. $278 \sim 284,2013$.

[3] Hongxin Wan, Yun Peng, "Clustering and Evaluation on Electronic Commerce Customers Based on Fuzzy Set", IJACT, Vol. 5, No. 3, pp. $199 \sim 206,2013$.

[4] Ling Liu, Zijiang Yang, "Improving Online Shopping Experience using Data Mining and Statistical Techniques", JCIT, Vol. 8, No. 6, pp. $466 \sim 474,2013$.

[5] Du Jin, "Application of "Internet of Things" in Electronic Commerce", JDCTA, Vol. 6, No. 8, pp. $222 \sim 230,2012$.

[6] Gebeyehu Belay Gebremeskel, Zhongshi He, Huazheng Zhu, "An Architecture of Intelligent Cloud Data Mining Platform for Optimization of Cloud Based Business Intelligence", JCIT, Vol. 8, No. 4, pp. $304 \sim 312,2013$.

[7] Xiaojun WU, "The Application of Neural Network Technology in Electronic Commerce Evaluation System", JDCTA, Vol. 5, No. 12, pp. $233 \sim 239,2011$.

[8] Shu-Meng Huang, "Adopting Data Mining Techniques on the Recommendations of Library Collections", RNIS, Volume 12, pp. $76 \sim 82,2013$.

[9] Bin Liu, "Research on Logistics Distribution Center Location Model and Evaluation Under Electronic Commerce", JDCTA, Vol. 7, No. 2, pp. $216 \sim 222,2013$.

[10] Yun-Peng Cai, "Construction Personalized Recommendation System Of E-Commerce Based On Variable Precision Rough Set", IJACT, Vol. 4, No. 11, pp. $125 \sim 131,2012$. 